\title{
Far-Infrared Study of the Charge Density Wave in Tetrathiofulvalene Tetracyanoquinodimethane (TTF-TCNQ)
}

\author{
Tanner, D. B.; Cummings, K. D.; Jacobsen, Claus Schelde
}

Published in:

Physical Review Letters

Link to article, DOI:

10.1103/PhysRevLett.47.597

Publication date:

1981

Document Version

Publisher's PDF, also known as Version of record

Link back to DTU Orbit

Citation (APA):

Tanner, D. B., Cummings, K. D., \& Jacobsen, C. S. (1981). Far-Infrared Study of the Charge Density Wave in Tetrathiofulvalene Tetracyanoquinodimethane (TTF-TCNQ). Physical Review Letters, 47(8), 597-600. https://doi.org/10.1103/PhysRevLett.47.597

\section{General rights}

Copyright and moral rights for the publications made accessible in the public portal are retained by the authors and/or other copyright owners and it is a condition of accessing publications that users recognise and abide by the legal requirements associated with these rights.

- Users may download and print one copy of any publication from the public portal for the purpose of private study or research.

- You may not further distribute the material or use it for any profit-making activity or commercial gain

- You may freely distribute the URL identifying the publication in the public portal 


\title{
Far-Infrared Study of the Charge Density Wave in Tetrathiafulvalene Tetracyanoquinodimethane (TTF-TCNQ)
}

D. B. Tanner and K. D. Cummings

Department of Physics, The Ohio State University, Columbus, Ohio 43210

and

C. S. Jacobsen

Physics Laboratory 3, Technical University of Denmark, DK-2800 Lyngby, Denmark (Received 27 March 1981)

\begin{abstract}
Detailed far-infrared measurements at temperatures from 25 to $300 \mathrm{~K}$ provide strong support for a charge-density-wave mechanism for the dc conductivity and microwave dielectric constant of tetrathiafulvalene tetracyanoquinodimethane (TTF-TCNQ). At low temperatures the charge-density wave is pinned at $40 \mathrm{~cm}^{-1}$ while at higher temperatures it appears at zero frequency. Values are obtained for the effective mass $\left(20 m^{*}\right)$ and lifetime $\left(1.6 \times 10^{-12} \mathrm{sec}\right.$ at $\left.60 \mathrm{~K}\right)$ of the charge-density wave.
\end{abstract}

PACS numbers: $78.30 . \mathrm{Jw}, 71.30 . \mathrm{th}, 72.15 . \mathrm{Nj}$

Despite considerable experimental and theoretical efforts over the past eight years, the properties of tetrathiafulvalene tetracyanoquinodimethane (TTF-TCNQ) remain controversial. The principal point of dispute is between single-particle models and models incorporating collective effects. The single-particle models explain the temperature dependence of the conductivity by scattering of the conduction electrons from phonons ${ }^{1,2}$ or other electrons. ${ }^{3}$ The collective picture assumes that the important $2 k_{\mathrm{F}}$ phonons are in equilibrium with the electrons rather than with the lattice. This assumption, an extreme case of phonon drag, leads to a sliding charge-densitywave (CDW) picture for the conductivity..$^{4-7}$

The importance of collective effects is supported by diffuse $x-$ ray $^{8}$ and neutron ${ }^{9}$ scattering measurements [which observe a three-dimensionally (3D) ordered CDW below $38 \mathrm{~K}$ and one-dimensional (1D) fluctuations up to $150 \mathrm{~K}$ ] and by conductivity measurements at high pressure ${ }^{10}$ (which show a drop in conductivity when the CDW becomes commensurate to the lattice). The CDW mechanism is, however, certainly not universally accepted at the present time. In this Letter we report the results of far-inf rared reflection measurements on TTF-TCNQ at temperatures between 25 and $300 \mathrm{~K}$. Our data indicate that near $60 \mathrm{~K}$ the dc conductivity of this material is dominated by a low-frequency mode which we interpret as a sliding charge-density wave. At temperatures below $38 \mathrm{~K}$ the CDW becomes pinned at relatively high frequencies $\left(\sim 40 \mathrm{~cm}^{-1}\right)$ where it is the major contributor to the microwave dielectric constant.

Far infrared (FIR) measurements at low temperatures are central to the single-particle ver- sus collective-mode issue since the latter model makes rather definite predictions about the farinfrared properties. ${ }^{11}$ At low temperatures, the $\mathrm{CDW}$ is pinned by $3 \mathrm{D}$ interactions or impurities and is observed as an FIR absorption peak. ${ }^{5}$ At higher temperatures, the CDW slides through the crystal, contributing a Drude-like term to the frequency-dependent conductivity,

$$
\sigma_{1}(\omega)=\sigma_{1 \mathrm{sp}}+\Omega_{p}{ }^{2} \tau_{c} / 4 \pi\left(1+\omega^{2} \tau_{c}^{2}\right),
$$

where $\sigma_{1 s p}$ is the single-particle contribution to the conductivity, $\tau_{c}$ is the collective-mode lifetime, and $\Omega_{p}=\left(4 \pi n e^{2} / M^{*}\right)^{1 / 2}$ with $n$ the carrier density ( 0.59 per molecule for TTF-TCNQ) and $M^{*}$ the CDW effective mass. Because the lifetime of the collective mode is very long, its contribution to the conductivity is only significant at very low frequencies, $\omega<1 / \tau_{c}$.

Polarized FIR reflectance measurements were made over 7 to $700 \mathrm{~cm}^{-1}$ using a Michelson interferometer in conjunction with a germanium bolometer detector operating at $1 \mathrm{~K}$. The exit aperture of the interferometer was imaged onto the sample by a large $(30-\mathrm{cm}-$ diam and $18-\mathrm{cm}-$ focal length) mirror and the reflected light was focused by an identical mirror into the entrance of the light pipe leading to the detector. The polarizer was a gold wire grid on polyethelene.

The sample was a mosaic of nine TTF-TCNQ crystals, each $\sim 1.2$ by $0.15 \mathrm{~cm}$ in size, mounted in an Air Products Helitran cryostat. One crystal had four electrical connections to its back surface to enable resistance measurements; the sample temperature was determined by this resistance. This calibration was necessary because room-temperature radiation heated the sample 
and prevented cooling below $25 \mathrm{~K}$. The mosaic intercepted more than $90 \%$ of the far-infrared beam and gave a signal-to-noise ratio in the reflectance exceeding 100 . The reflectance at a given temperature, $R(T)$, was determined from four measurements: $R(T)=\left[S_{1}(T) / S_{1}(300)\right]\left[S_{2}(300) /\right.$ $S_{2}(\mathrm{Au})$ ]. Here $S_{1}(T)$ is the signal from the mosaic at temperature $T ; S_{1}(300)$ is the signal at room temperature; $S_{2}(300)$ is the room-temperature signal from a mosaic; and $S_{2}(\mathrm{Au})$ is the signal from the same mosaic after coating with gold.

Figure 1 shows the $\vec{b}$-axis polarized reflectance, $R(\omega)$, of TTF-TCNQ at five temperatures between 25 and $300 \mathrm{~K}$. At room temperature, ${ }^{12} R(\omega) \rightarrow 1$ at $\omega \sim 0$. The slope of $R(\omega)$ is negative at low frequencies, as expected for a good conductor. At higher frequencies $R(\omega) \sim 0.75$. At 160 and $60 \mathrm{~K}$, $R(\omega)$ is qualitatively similar; its magnitude increases as the temperature is lowered while the negative slope at low frequencies [with $R(\omega) \rightarrow 1$ at $\omega \sim 0$ ] remains. At $25 \mathrm{~K}$ the reflectance is profoundly different: It has positive slope at low frequencies and a maximum around $40-80 \mathrm{~cm}^{-1}$ followed by deep minimum at $120 \mathrm{~cm}^{-1}$. The $34-\mathrm{K}$ reflectance is intermediate between the $25-\mathrm{K}$ and

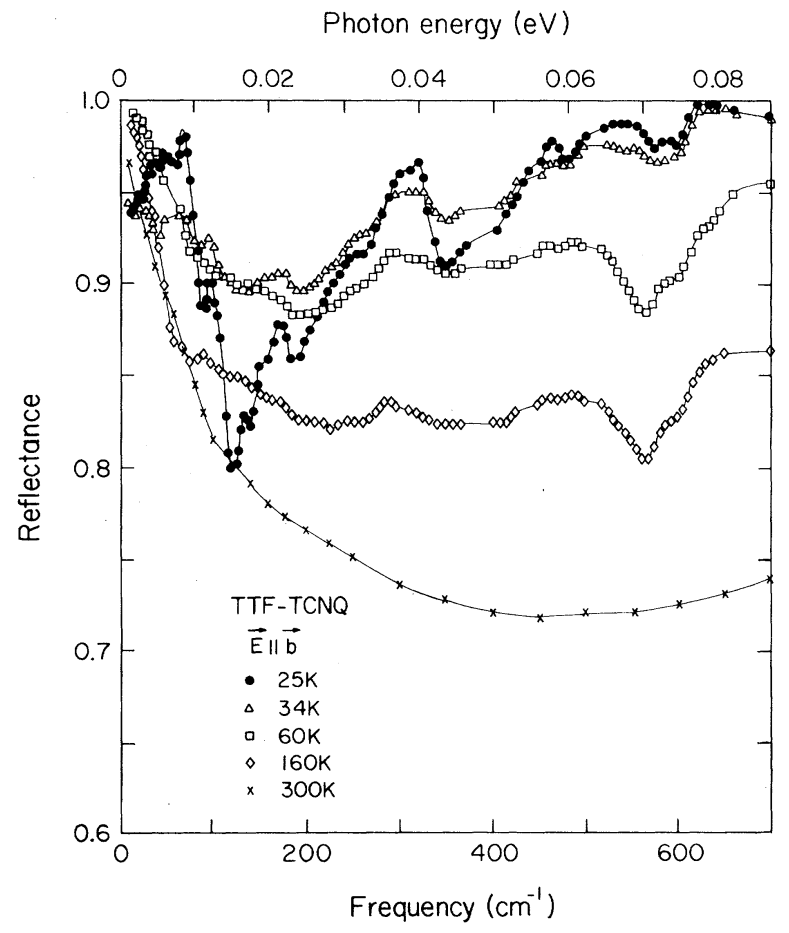

FIG. 1. Reflectance of TTF-TCNQ for electric field along the $\vec{b}$-axis. Note the suppressed zero. Lines are drawn as a guide to the eye. the $60-\mathrm{K}$ data.

We have performed a Kramers-Kronig analysis of the reflectance data. ${ }^{13}$ At frequencies about $700 \mathrm{~cm}^{-1}$ the reflectance was extrapolated smoothly from the measured data, with a transition ${ }^{12}$ at $1600 \mathrm{~cm}^{-1}$ to values calculated from the near-infrared reflectance data of Bright, Garito, and Heeger. ${ }^{14}$ The reflectance at low frequencies was smoothly extrapolated using the Hagen-Reubens relation in the conducting regime and was assumed constant at lower temperatures..$^{13}$

Figure 2 shows the $\vec{b}$-axis frequency-dependent conductivity of TTF-TCNQ. The low-frequency conductivity is in complete agreement with the dc conductivity of these "ordinary" crystals. ${ }^{15}$ It rises from $500 \Omega^{-1} \mathrm{~cm}^{-1}$ at room temperature to $5000 \Omega^{-1} \mathrm{~cm}^{-1}$ at $60 \mathrm{~K}$ and then falls to around $200 \Omega^{-1} \mathrm{~cm}^{-1}$ at $34 \mathrm{~K}$ and is small at $25 \mathrm{~K}$. We stress that the low-frequency conductivity is not affected in any significant way by the choice of extrapolation procedure. Assuming constant reflectance below our lowest data point reduced the value of the $60-\mathrm{K}$ conductivity at that point by $20 \%$ or so; the narrow structure at low frequency, however, still remained. At $25 \mathrm{~K}$ the conductivity has a strong peak at $40 \mathrm{~cm}^{-1}$. This feature is reduced in strength and broadened in the $34-\mathrm{K}$ data and is not present at $60 \mathrm{~K}$ and above. There is other structure at higher frequencies, most notably an intense peak at $290 \mathrm{~cm}^{-1}$.

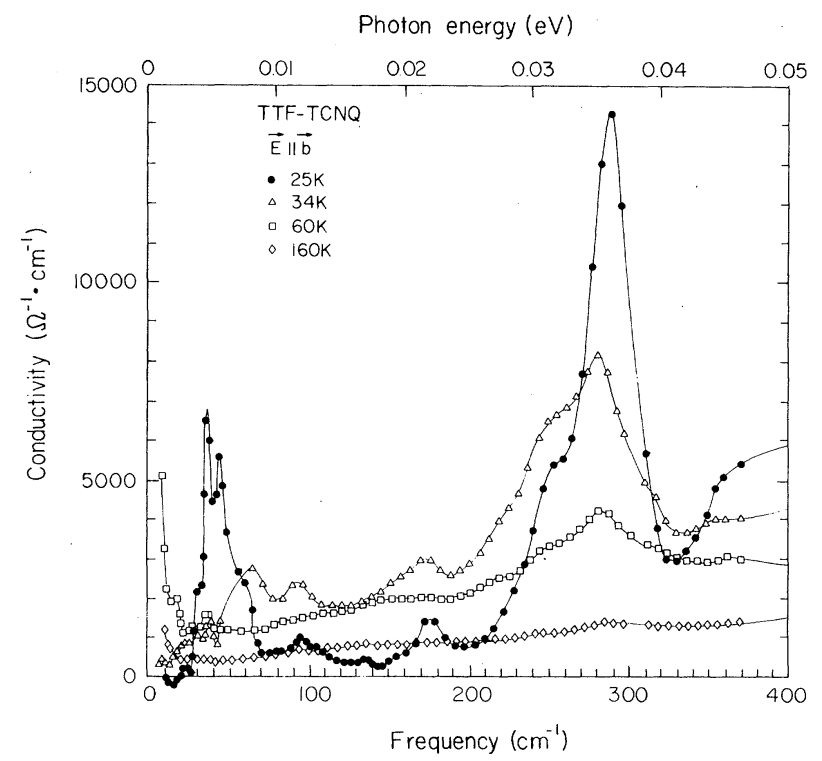

FIG. 2. Frequency-dependent $\vec{b}$-axis conductivity of TTF-TCNQ at four temperatures: $25,34,60$, and $160 \mathrm{~K}$. 
Figure 3 shows the real part of the $\vec{b}$-axis dielectric function of TTF-TCNQ. The low-frequency FIR data are in excellent numerical agreement with microwave dielectric constant measurements. We find $\epsilon_{1}(0)=3200$ at $25 \mathrm{~K}$, within $10 \%$ of the values of Gunning et al. ${ }^{16}$ at these temperatures. At $34 \mathrm{~K}$, the dielectric constant has increased to 6000 while it is negative and very large in magnitude in the highly conducting state.

The $40-\mathrm{cm}^{-1}$ feature in the $25-\mathrm{K}$ conductivity has a large oscillator strength. We identify this feature as the phase mode of the pinned CDW in TTF-TCNQ. The effective mass $M^{*}$ of the pinned charge-density wave can be estimated from the oscillator-strength sum rule,

$$
\int_{0}^{80} \sigma_{1}(\omega) d \omega=\frac{1}{2} \pi\left(n e^{2} / M^{*}\right)=\frac{1}{8} \Omega_{\rho}^{2} .
$$

The upper limit of the integral is taken as $80 \mathrm{~cm}^{-1}$, where the high-frequency wing of the peak has dropped into the baseline. We obtain $M^{*}=60 m_{e}$ $=20 m *\left(m_{e}\right.$ is the free-electron mass and $m *$ is the ordinary band mass).

While small, this effective mass is consistent with microwave dielectric constant data. According to Lee, Rice, and Anderson there are three contributions to the static dielectric constant in a

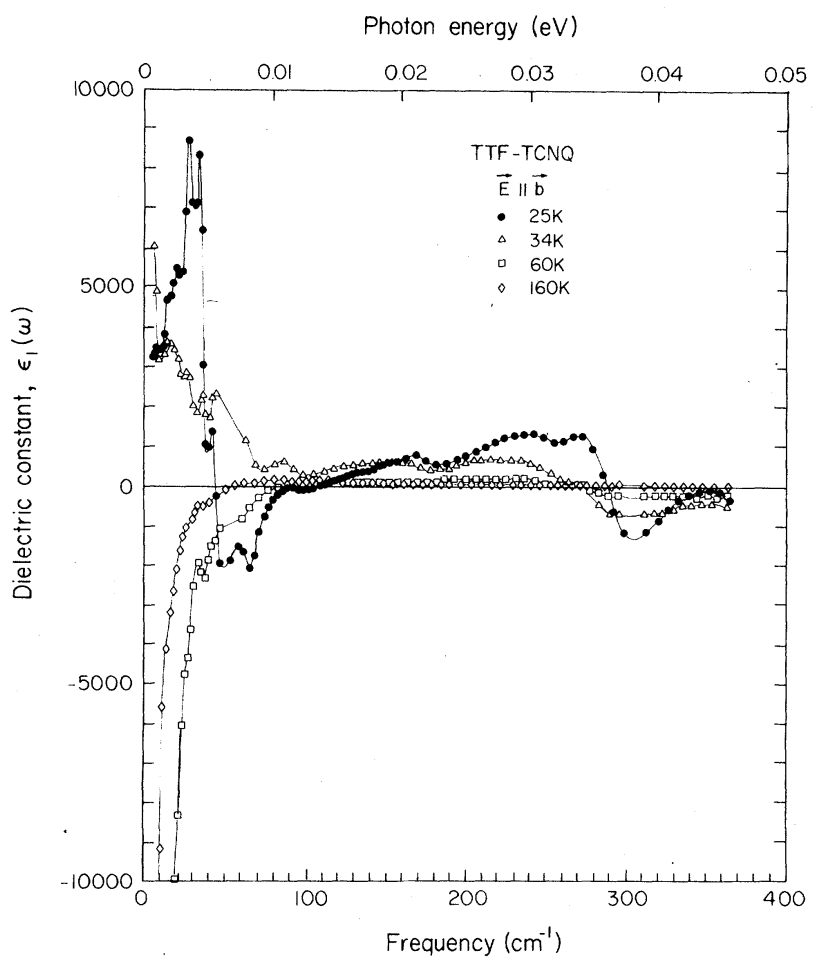

FIG. 3. Real part of the $\vec{b}$-axis dielectric function of TTF-TCNQ.
CDW system,

$$
\epsilon_{1}=\epsilon_{\infty}+\frac{2}{3} \omega_{p}^{2} /(2 \Delta)^{2}+\Omega_{p}^{2} / \omega_{T}^{2},
$$

where $\epsilon_{\infty}$ is the high-frequency dielectric constant, $\omega_{p}$ is the conduction-electron plasma frequency, $2 \Delta$ is the Peierls electronic energy gap, and $\omega_{T}$ is the CDW pinning frequency. Using the above estimate of the CDW effective mass, we obtain $\Omega_{p}=2000 \mathrm{~cm}^{-1}$; our conductivity data indicate that $\omega_{T}=40 \mathrm{~cm}^{-1}$. The CDW contribution to the dielectric constant is then 2700. At frequencies well above the pinning frequency only the first two terms on the right-hand side of Eq. (3) contribute to $\epsilon_{1}$. At $25 \mathrm{~K}, \epsilon_{1} \sim 600$ for $100 \mathrm{~cm}^{-1}<\omega$ $<200 \mathrm{~cm}^{-1}$, a reasonable value for the singleparticle contribution to $\epsilon_{1}$. The total dielectric constant is then predicted to be 3300 ; experimentally ${ }^{16}$ it is between 3000 and 3500 .

At $60 \mathrm{~K}$, the $\mathrm{CDW}$ is at zero frequency where its finite dc conductivity drives $\epsilon_{1}(\omega)$ negative at low frequencies. [Note that $\epsilon_{1}(\omega)>0$ above 100 $\mathrm{cm}^{-1}$.] The zero crossing occurs at a screened CDW plasma frequency $\Omega_{p} / \sqrt{\epsilon_{H}}$ where $\epsilon_{H}$ is the value of $\epsilon_{1}(\omega)$ above the zero. From the data in Fig. 3 we obtain $\Omega_{p}=1000 \mathrm{~cm}^{-1}$ at $60 \mathrm{~K}$ and $\Omega_{p}$ $=500 \mathrm{~cm}^{-1}$ at $160 \mathrm{~K}$. The CDW appears to lose oscillator strength with increasing temperature.

With $\Omega_{p}$ known, the lifetime of the collective mode may be estimated. We use Eq. (1) [but put $\left.\sigma_{1 \mathrm{sp}}(0)=0\right]$ and take $5000 \Omega^{-1} \mathrm{~cm}^{-1}$ for the $60-\mathrm{K}$ conductivity. We then obtain $\tau_{c}=1.6 \times 10^{-12} \mathrm{sec}$ $\left(1 / \tau_{c}=4 \mathrm{~cm}^{-1}\right)$ in agreement with the width of the narrow low-frequency peak in $\sigma_{1}(\omega)$ at $60 \mathrm{~K}$ in Fig. 2. In contrast the single-particle lifetime ${ }^{14}$ is $\tau_{\mathrm{sp}} \sim 6 \times 10^{-15} \mathrm{sec}$.

The $25-\mathrm{K}$ results for the CDW mass and pinning frequency are significantly different from those of earlier low-temperature FIR studies of TTFTCNQ single crystals by Coleman et al..$^{17}$ and by Eldridge and Bates. ${ }^{18}$ (We note that the data in Fig. 2 resemble early results on sublimed thin films of TTF-TCNQ, although the magnitude of the conductivity was much lower and the frequency of the structure identified as the pinned mode was higher. ${ }^{19}$ ) The polarized reflectance data of Coleman et al.$^{17}$ did not extend above $100 \mathrm{~cm}^{-1}$ and so did not include the $125-\mathrm{cm}^{-1}$ reflectance minimum seen in our $25-\mathrm{K}$ data. The magnitude of the reflectance was also lower than ours (from depolarization in the light pipes that they used). In their fit to a model CDW dielectric function they assumed the reflectance minimum to be below rather than above their frequency range and therefore obtained a low value for the pinning frequen- 
cy and, consequently, too large a CDW effective mass.

Eldridge and Bates ${ }^{18}$ used a TTF-TCNQ crystal as a far-infrared detector. If $\alpha d \ll 1$ ( $\alpha$ is the absorption coefficient; $d$ the thickness) their signal would be proportional to $\alpha$; otherwise it would saturate to $1-R$ ( $R$ is the reflectance). Our Kramers-Kronig analysis indicates that $\alpha>10^{4} \mathrm{~cm}^{-1}$ at $25 \mathrm{~K}$ for frequencies above $30 \mathrm{~cm}^{-1}$ so that their samples, with $d>3 \times 10^{-3} \mathrm{~cm}$, were in the limit $\alpha d \gg 1$; hence the signal was saturated at $1-R$. Their data closely resemble our results for $1-R$ at frequencies below $300 \mathrm{~cm}^{-1}$.

In summary, our data support the picture of TTF-TCNQ that attributes its unusual dc conductivity and microwave dielectric constant to collective effects associated with the charge-density wave. TTF-TCNQ is neither a metal above $58 \mathrm{~K}$ nor an ordinary semiconductor below $38 \mathrm{~K}$.

The high-quality crystals used in this study were grown by I. Johannsen and L. Groth-Andersen. The work was supported by the Danish Natural Sciences Research Council and the NATO Research Grants Program.

${ }^{1}$ H. Gutfreund and M. Weger, Phys. Rev. B 16, 1753 (1977); M. Weger, M. Kaveh, and H. Gutfreund; Solid State Commun. 32, 323 (1979).

${ }^{2}$ E. M. Conwell, Phys. Rev. Lett. 39, 777 (1977), and Phys. Rev. B 22, 1761 (1980).

${ }^{3}$ P. E. Seiden and D. Cabib, Phys. Rev. B 13,1846 (1976).

${ }^{4}$ H. Fröhlich, Proc. Roy. Soc. London A 223, 296 (1954).

${ }^{5}$ P. A. Lee, T. M. Rice, and P. W. Anderson, Solid State Commun. 14, 703 (1974).

${ }^{6} \mathrm{D}$. Allender, J. W. Bray, and J. Bardeen, Phys. Rev. B 9,119 (1974).

${ }^{7}$ A. J. Heeger, M. Weger, and M. Kaveh, in Quasi
One-Dimensional Conductors $I$, Lecture Notes in Physics, Vol. 95, edited by S. Barišić, A. Bjeliš, J. R. Cooper, and B. Leontić (Springer-Verlag, New York, 1979), p. 316.

${ }^{8}$ F. Denoyer, R. Comes, A. F. Garito, and A. J. Heeger, Phys. Rev. Lett. 35, 445 (1975); S. Kagoshima, H. Anzai, K. Kajimura, and T. Ishigoro, J. Phys. Soc. Jpn. 39, 1143 (1975).

${ }^{9}$ R. Comes, S. M. Shapiro, G. Shirane, A. F. Garito, and A. J. Heeger, Phys. Rev. Lett. 35, 1518 (1975), and Phys. Rev. B 14, 2376 (1976).

${ }^{10}$ R. H. Friend, M. Miljak, and D. Jerome, Phys. Rev. Lett. 40, 1048 (1978); A. Andrieux, H. J. Shulz, D. Jerome, and K. Bechgaard, Phys. Rev. Lett. 43 , 227 (1979).

${ }^{11} \mathrm{~A}$. J. Heeger, in Highly Conducting One-Dimensional Solids, edited by J. T. Devreese, R. P. Evrard, and V. E. van Doren (Plenum, New York, 1979), p. 69.

${ }^{12} \mathrm{C}$. S. Jacobsen, in Quasi One-Dimensional Conductors $I$, Lecture Notes in Physics, Vol. 95, edited by S. Barišić, A. Bjelišs, J. R. Cooper, and B. Leontić (SpringerVerlag, New York, 1979), p. 223.

${ }^{13}$ Kramers-Kronig relations, extrapolation procedures, and sum rules are discussed in detail by $\mathrm{F}$. Wooten, Optical Properties of Solids (Academic, New York, 1972).

${ }^{14}$ A. A. Bright, A. F. Garito, and A. J. Heeger, Phys. Rev. B 10, 1328 (1975).

${ }^{15}$ M. J. Cohen, L. B. Coleman, A. F. Garito, and A. J. Heeger, Phys. Rev. B 13, 5111 (1976); L. B. Coleman, Ph.D. thesis, University of Pennsylvania, 1975 (unpublished).

${ }^{16}$ W. J. Gunning, S. K. Khanna, A. F. Garito, and A. J. Heeger, Solid State Commun. 21, 765 (1977); W. J. Gunning and A. J. Heeger, Phys. Status Solidi (b) $\underline{95}$, 433 (1979)

${ }^{17}$ L. B. Coleman, C. R. Fincher, Jr., A. F. Garito, and A. J. Heeger, Phys. Status Solidi (b) $\underline{75}, 239$ (1976).

${ }^{18} \mathrm{~J}$. E. Eldridge and F. E. Bates, Solid State Commun. 30, 195 (1979); see also J. E. Eldridge, Solid State Commun. 19, 607 (1976), and 21, 737 (1977), and 26 , 243 (1978)

${ }^{19}$ D. B. Tanner, C. S. Jacobsen, A. F. Garito, and A. J. Heeger, Phys. Rev. B 13, 3381 (1976). 